\title{
Miradas de la naturaleza de la ciencia y la tecnología desde la perspectiva CTSA de maestros en escuelas normales
}

\author{
Tuay Sigua Rosa Nidia', Pérez Mesa María Rocio², Porras Yair Alexander ${ }^{3}$
}

Categoría 2. Trabajo de investigación (en proceso)

\section{Resumen}

El estudio de temáticas referentes a la manera en que los profesores en ejercicio, conciben la forma como funciona la ciencia y la tecnología (CyT) en el mundo actual, cuál es la naturaleza de la CyT y las relaciones entre la ciencia, la tecnología, la sociedad y el ambiente (CTSA) como componentes centrales de la alfabetización científica y tecnológica, se constituye en una oportunidad para construir un espacio de articulación con 3 Escuelas Normales Superiores (ENS) que superen el carácter de asesoría que ha sido tradicional en la relación Universidad -Escuela permitiendo desarrollar a través de la investigación un espacio de construcción colectiva y sostenible.

\section{Palabras clave}

Formación permanente de docentes, Naturaleza de ciencia y tecnología, enseñanza de ciencia y tecnología, alfabetización de ciencia y tecnología,

\section{Objetivo}

Coadyuvar a la comprensión sobre NdCyT de profesores de 3 Escuelas Normales Superiores participantes en el proyecto PARES a través de la construcción de estrategias didácticas teniendo en cuenta los contextos regionales.

\section{Marco Teórico}

Uno de los campos de estudio que en los últimos años ha concentrado la atención de investigadores con referencia a la formación inicial y continua de docentes está referido en la importancia de comprender las complejas

\footnotetext{
1 Universidad Pedagógica Nacional. rtuay@pedagogica.edu.co

2 Universidad Pedagógica Nacional.mperez@pedagogica.edu.co

3 Universidad Pedagógica Nacional. yporras@pedagogica.edu.co
} 
Revista Tecné, Episteme y Didaxis: TED. Año 2014, Número Extraordinario. ISSN Impreso: 0121-3814, ISSN web: 2323-0126 Memorias, Sexto Congreso Internacional sobre Formación de Profesores de Ciencias. 08 al 10 de octubre de 2014, Bogotá

relaciones entre la Ciencia, la Tecnología, la Sociedad y el Ambiente (CTSA). En este sentido, la innovación filosófica, metodológica y curricular que constituyen las relaciones CTSA ha promovido una serie de investigaciones que plantean la necesidad de una transformación radical del currículo en todos los niveles de enseñanza, con el objeto de formar, no sólo en conocimientos sino en valores que favorezcan la participación ciudadana responsable y democrática en la evaluación y el control de las implicaciones sociales de la ciencia y la tecnología (Waks, 1996).

De acuerdo con algunas investigaciones (Acevedo, 1996, Callejas y Mendoza, 2010) ciertas dificultades que prevalecen para la incorporación de las relaciones CTSA en la práctica educativa, se deben a las visiones deformadas sobre la ciencia, el trabajo científico y la tecnología que tienen los profesores de enseñanza secundaria en ejercicio. Así mismo, Vazquez, Manassero (2012) en su propuesta de trabajo plantean que

Hoy se acepta que la alfabetización en CyT está formada por dos componentes: i) los conceptos y teorías de la CyT (los tradicionales conceptos, hechos y principios "de" ciencia y tecnología que forman el cuerpo de leyes y teorías científicas); ii) los innovadores conocimientos "sobre" la ciencia y tecnología, que permiten comprender como funcionan la ciencia y tecnología (CyT). Este segundo componente de la alfabetización se denomina naturaleza de la ciencia y tecnología (NdCyT) y constituye el reto más innovador y arduo para los profesores de ciencias. En los últimos años ha sido incorporado de diversas maneras en los currículos escolares de numerosos países, y nunca antes se había planteado con la extensión y profundidad actuales. (pág. 2)

En este sentido, es necesario ahondar en el estudio de temáticas referentes a la manera en que los profesores en ejercicio, conciben la forma como funcionan la ciencia y la tecnología (CyT) en el mundo actual, cuál es la naturaleza de la CyT y las relaciones entre la ciencia, la tecnología, la sociedad y el ambiente (CTSA) como componentes centrales de la alfabetización científica y tecnológica. De igual forma, la presente propuesta se plantea la necesidad de construir de manera conjunta la realización de actividades reflexivas que aporten a los procesos de enseñanza y aprendizaje en este ámbito. 
Revista Tecné, Episteme y Didaxis: TED. Año 2014, Número Extraordinario. ISSN Impreso: 0121-3814, ISSN web: 2323-0126 Memorias, Sexto Congreso Internacional sobre Formación de Profesores de Ciencias. 08 al 10 de octubre de 2014, Bogotá

La comprensión de la Naturaleza de la Ciencia y la Tecnología (NdCyT) es considerada por los especialistas un componente central de la alfabetización científica para todos y como tal se incorpora en los contenidos de los currículos escolares; además, la investigación sobre NdCyT constituye una línea innovadora en la investigación didáctica, en la enseñanza y en el aprendizaje de Ciencia y Tecnología (CyT). Sin embargo, la literatura especializada informa que estudiantes y profesores no logran comprender bien la NdCyT, de modo que algunas investigaciones recientes con profesores y estudiantes se han dirigido a aclarar la efectividad de diversas metodologías para mejorar la enseñanza y el aprendizaje de la NdCyT, cuyos resultados revelan que la efectividad educativa tiene dos condiciones clave: el carácter explícito de la enseñanza y la realización de actividades reflexivas sobre $\mathrm{NdCyT}$.

Esta investigación afronta el problema educativo de enseñar con calidad la naturaleza de la ciencia y la tecnología (NdCyT), es decir, las cuestiones acerca de cómo la ciencia y la tecnología (CyT) validan su conocimiento y cómo funcionan en el mundo actual. NdCyT engloba aspectos de epistemología y sociología de CyT y las relaciones entre la ciencia, la tecnología, la sociedad y el ambiente (CTSA), unos contenidos complejos e innovadores, y poco populares en la educación científica. Mejorar la calidad de la enseñanza sobre NdCyT a través de la intervención en la formación continuada de docentes de Escuelas Normales Superiores participantes en el proyecto PARES es deseable pues contribuirá a alcanzar mejor calidad del aprendizaje sobre NdCyT por medio de del desarrollo de estrategias didácticas.

El proyecto PARES es una iniciativa que busca aportar a la puesta en marcha y consolidación de políticas y programas de regionalización de la Universidad Pedagógica Nacional, se espera que el modelo permita generar las condiciones para pensar la regionalización con las unidades académicas y administrativas de la institución en espacios de encuentro, debate y construcción colectiva a partir de la construcción conjunta de escenarios de desarrollo regional en los ámbitos de: a) formación inicial y continuada de educadores, a través de propuestas de innovación pedagógica que den respuesta a las necesidades de los diversos territorios; b) investigación, basada en diálogos de saberes en torno a la pedagogía y el estímulo a la apropiación social del conocimiento; c) proyección social, a través del desarrollo colaborativo de proyectos educativos dentro y fuera de la escuela y con diversos actores. 
Revista Tecné, Episteme y Didaxis: TED. Año 2014, Número Extraordinario. ISSN Impreso: 0121-3814, ISSN web: 2323-0126 Memorias, Sexto Congreso Internacional sobre Formación de Profesores de Ciencias. 08 al 10 de octubre de 2014, Bogotá

Las preguntas de investigación que se plantean en este trabajo de investigación, son las siguientes:

¿Qué tipo de representaciones y actitudes sobre la naturaleza de la ciencia y la tecnología desde la perspectiva CTSA son elicitadas por los profesores de ciencias de Escuelas Normales Superiores participantes en el proyecto PARES a través de la aplicación del COCTS?

¿Qué aspectos pueden configurar el diseño de una unidad didáctica para la enseñanza de la NdCyT desde la perspectiva CTSA acorde con los contextos regionales en las que se encuentran las Escuelas Normales Superiores participantes en el proyecto PARES?

¿Qué estrategias se pueden construir para vincular a las Escuelas Normales Superiores en redes de investigación sobre CTSA?

\section{Metodología}

La investigación propone una metodología cualitativa que vislumbre las emergencias propias de la interacción entre los miembros de la comunidad educativa, con el objeto de iniciar una discusión sobre las implicaciones sociales, culturales, pedagógicas y didácticas que tiene el estudio de dichas interacciones, desde una mirada crítica del mundo. Se plantean tres etapas para el desarrollo de la investigación:

Etapa I: Corresponde a la consolidación de la situación problema estudiada a partir del reconocimiento de sus propiedades esenciales. En este proyecto de investigación, concierne a la revisión de los resultados de investigación de los proyectos de las vigencias anteriores. Como los análisis de las preguntas del COCTS relacionadas con naturaleza de las ciencias.

Etapa II: El trabajo de campo que hace referencia al periodo de recolección y organización de los datos. Para efecto de esta investigación se refiere a la revisión de las relaciones CTSA en el quehacer de los docentes de las Escuelas Normales a través de la aplicación del cuestionario COCTS. Los docentes de las 3 Escuelas Normales participantes forman parte del proyecto PARES y manifestaron de manera voluntaria participar en el proyecto después de una convocatoria abierta a todos los docentes. La aplicación de los cuestionarios se hará en situ por los investigadores del proyecto. 
Revista Tecné, Episteme y Didaxis: TED. Año 2014, Número Extraordinario. ISSN Impreso: 0121-3814, ISSN web: 2323-0126 Memorias, Sexto Congreso Internacional sobre Formación de Profesores de Ciencias. 08 al 10 de octubre de 2014, Bogotá

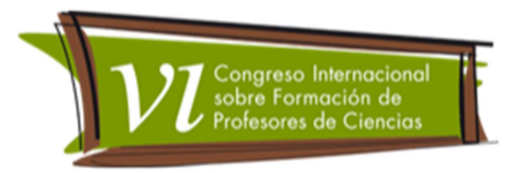

Etapa III: En ésta se hace alusión a la identificación de los patrones que organizan la situación problema estudiada. Al respecto, el análisis, la interpretación y la conceptualización de la información, permite el establecimiento de las correlaciones entre las diferentes fuentes documentales, que aportarán a la Construcción colectiva de las estrategias didácticas para el abordaje de la Naturaleza de Ciencia y Tecnología desde la perspectiva CTSA.

Esta investigación de tipo descriptivo intenta aproximarse al conocimiento de las situaciones, costumbres y actitudes predominantes a través de la descripción exacta de las actividades, objetos, procesos y personas. Su meta no se limita a la recolección de datos, sino a la predicción e identificación de las relaciones que existen entre dos o más variables. Los investigadores dan tratamiento cuidadoso a la información y luego analizan minuciosamente los resultados, a fin de extraer generalizaciones significativas que contribuyan al conocimiento en este caso de las representaciones y actitudes que presentan los docentes de las Escuelas Normales.

\section{Resultados}

La relación de docentes participantes en el proyecto de investigación se muestra en la Tabla 1.

Tabla 1. Profesores de Escuelas Normales participantes en el proyecto

\begin{tabular}{|c|c|c|c|c|}
\hline $\begin{array}{rr} & \begin{array}{r}\text { Escuela Normal } \\
\text { Superior }\end{array} \\
\text { Género } & \end{array}$ & $\begin{array}{c}\text { Maria Inmaculada de } \\
\text { Arauca }\end{array}$ & $\begin{array}{c}\text { San Juan del } \\
\text { César }\end{array}$ & Acacías & TOTAL \\
\hline Femenino & 6 & 7 & 5 & 18 \\
\hline Masculino & 4 & 6 & 5 & 15 \\
\hline TOTAL & 10 & 13 & 10 & 33 \\
\hline
\end{tabular}

En esta instancia de la investigación se están aplicando los cuestionarios COCTS a los docentes de las Escuelas Normales y se espera tener resultados definitivos cuando el Congreso de Formación de profesores se lleve a cabo. La construcción de instrumentos se hará a partir de las cuestiones del COCTS, para adaptarlos a los objetivos y los contenidos de la intervención didáctica que será construida en cada institución.

A partir de los resultados y teniendo en cuenta Las investigaciones realizadas por el grupo de investigación se busca aportar a la comprensión de NdCyT en una 
Revista Tecné, Episteme y Didaxis: TED. Año 2014, Número Extraordinario. ISSN Impreso: 0121-3814, ISSN web: 2323-0126 Memorias, Sexto Congreso Internacional sobre Formación de Profesores de Ciencias. 08 al 10 de octubre de 2014, Bogotá

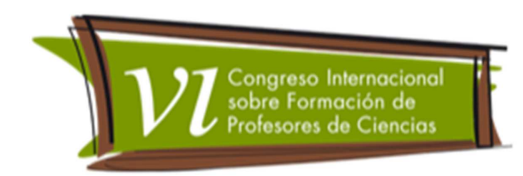

variedad de contextos y metodologías apuntando a que el tratamiento intencional y explícito de NdCyT junto con actividades de reflexión se constituya en un método eficaz para mejorar la comprensión de la NdCyT. En consecuencia, esta investigación trata de validar la efectividad de este punto de vista, ampliando la esfera de influencia a los profesores de las 3 Escuelas Normales Superiores y también a situaciones reales de aula utilizando procedimientos cuantitativos complementados con otros cualitativos para construir unidades didácticas, añadiendo los complementos adecuados de recursos y actividades.

\section{Conclusiones}

Los procesos de formación continua de docentes deben responder a los retos que plantea las relaciones del ser humano con otros, el ambiente y sus transformaciones incluyendo una visión integral y de totalidad de lo humano y de los procesos educativos, propiciando de esta manera una cultura de cambio y mejor disposición frente a la Ciencia, la Tecnología, la Sociedad y el Ambiente.

La Formulación de estrategias didácticas innovadoras que viabilicen la incorporación de la NdCyT desde la perspectiva CTSA en las Escuelas Normales Superiores desde una construcción colectiva que contemple los contextos regionales y fortalezca el dialogo de saberes permitirá el intercambio de conocimientos desde dos perspectivas: La relación vinculante entre la Universidad Pedagógica Nacional y las Escuelas Normales Superiores y también la relación entre los grupos de investigación en el marco de redes CTS.

Se busca la articulación de redes de investigación que permita el intercambio de conocimientos y experiencias entre los equipos y participantes del proceso investigativo que aporten al fortalecimiento y la consolidación de las redes y la dinamización del conocimiento acorde con las realidades educativas.

\section{Referencias bibliográficas}

Acevedo, J. A. 1996. La Tecnología en las Relaciones CTS. Una Aproximación al Tema. Enseñanza de las Ciencias, 14 (1), pp. 35-44.

Callejas, M. y Mendoza, E. (2010). Diferencias en la comprensión de la NdCyT entre profesores en formación que inician la universidad y los que finalizan su grado. En: Bennássar, A., Vázquez, A., Manassero, M, A., y García-Carmona, A (coords). Ciencia, Tecnología y Sociedad en Iberoamérica: Una 
Revista Tecné, Episteme y Didaxis: TED. Año 2014, Número

Extraordinario. ISSN Impreso: 0121-3814, ISSN web: 2323-0126

Memorias, Sexto Congreso Internacional sobre Formación de Profesores de Ciencias. 08 al 10 de octubre de 2014, Bogotá

Evaluación de la Comprensión de la Naturaleza de Ciencia y Tecnología. Madrid: Centro de Altos Estudios Universitarios (OEI).

Tuay y otros (2012). Evaluación de las representaciones y actitudes relacionadas con la ciencia, la tecnología, la sociedad y el ambiente en la Universidad Pedagógica Nacional. Ponencia en VII Seminario Iberoamericano CTS. Disponible en http://www.oei.es/seminarioctsm/bbdd_comunicaciones_autor.php?pageNum_co municaciones=15\&totalRows_comunicaciones=166\&autor_principal=. Revisado enero 2014.

Vázquez, A \& Manassero, M. (2012. La selección de contenidos para enseñar naturaleza de la ciencia y tecnología (parte 1): Una revisión de las aportaciones de la investigación didáctica. Revista Eureka sobre enseñanza y divulgación de la ciencia, 9(1), pp. 2-31.

Waks, L.J. (1996). Las relaciones escuela-comunidad y su influencia en la educación en valores en CTS. En A. Alonso, I. Ayestarán y N. Ursúa (Eds.): Para comprender Ciencia, Tecnología y Sociedad, pp. 35-47. Estella: EVD 